\title{
Single-electron transport driven by surface acoustic waves: moving quantum dots versus short barriers
}

\author{
P. Utko, ${ }^{1}$ J. Bindslev Hansen,${ }^{2}$ P. E. Lindelof, ${ }^{1}$ C. B. Sørensen, ${ }^{1}$ \\ and K. Gloos ${ }^{3}$ \\ ${ }^{1}$ Nano-Science Center and Niels Bohr Institute, University of Copenhagen, \\ Universitetsparken 5, DK-2100 Copenhagen, Denmark \\ ${ }^{2}$ Department of Physics, Technical University of Denmark, \\ DK-2800 Lyngby, Denmark \\ ${ }^{3}$ Wihuri Physical Laboratory, Department of Physics, University of Turku, \\ FIN-20014 Turku, Finland
}

(Received: March 24, 2006)

We have investigated the response of the acoustoelectric current driven by a surface-acoustic wave through a quantum point contact in the closed-channel regime. Under proper conditions, the current develops plateaus at integer multiples of ef when the frequency $f$ of the surface-acoustic wave or the gate voltage $V_{g}$ of the point contact is varied. A pronounced $1.1 \mathrm{MHz}$ beat period of the current indicates that the interference of the surface-acoustic wave with reflected waves matters. This is supported by the results obtained after a second independent beam of surface-acoustic wave was added, traveling in opposite direction. We have found that two sub-intervals can be distinguished within the $1.1 \mathrm{MHz}$ modulation period, where two different sets of plateaus dominate the acoustoelectric-current versus gate-voltage characteristics. In some cases, both types of quantized steps appeared simultaneously, though at different current values, as if they were superposed on each other. Their presence could result from two independent quantization mechanisms for the acoustoelectric current. We point out that short potential barriers determining the properties of our nominally long constrictions could lead to an additional quantization mechanism, independent from those described in the standard model of 'moving quantum dots'.

PACS numbers: 73.23.-b, 72.50.+b, 73.21.La. 


\section{P. Utko et al.}

\section{INTRODUCTION}

Single-electron transport through a quantum-point contact (QPC), driven by a surface-acoustic wave (SAW), is considered to be an attractive method towards a quantum standard of electrical current. ${ }^{[12}$ Such a standard would close the quantum-metrological triangle of electrical units of current, voltage, and resistance, and allow us to determine the electron charge $e$ and the Planck constant $h . \sqrt[3,4]{6}$ The SAW-based approach offers much higher operational frequencies (several $1 \mathrm{GHz})$ and output currents $(\sim 1 \mathrm{nA})$ than other types of electron pumps, $\frac{[5] 6 / 7 / 8}{,}$ which are limited to frequencies of order of $10 \mathrm{MHz}$ and output currents of about $10 \mathrm{pA}$. However, the low accuracy $9|10| 11|12| 13|14| 15]$ of the SAW pumps $(\sim 100 \mathrm{ppm})$ still prevents their metrological applications.

The SAW-driven single-electron devices have also been considered for other purposes. Foden et al. ${ }^{[16}$ suggested to use them as a key component of a single-photon source. In such a scheme, the SAW propagates along a lateral n-i-p junction. Its dynamic potential captures electrons in the n-type region, from the two-dimensional electron gas (2DEG) reservoir at the entrance to a QPC. Single electrons per SAW cycle are then transferred across the point contact and injected into the p-type region. They recombine there with holes, emitting a single photon per injected electron. In another proposal, Barnes et al. $\frac{17 \mid 18}{18}$ suggested to use spins of the single electrons trapped in the SAW minima as qubits for quantum-computation applications. They demonstrated theoretically the feasibility of such an approach for one- and two-qubit operations. This would involve a proper design of adjacent channels for the SAW-driven electrons and a pattern of magnetic and non-magnetic surface gates.

Qualitatively, the SAW pumps operate as follows. An interdigital transducer (IDT) generates - via the piezoelectric effect - elastic waves on the surface of a gallium arsenide sample that contains a 2DEG beneath its surface. Due to piezoelectricity, the mechanical component of the SAW is accompanied by an electrostatic potential which can induce a current flow across a closed quantum point contact. Shilton et al. ${ }^{[12}$ suggested that electrons are trapped in the 'moving quantum dots', local minima of the dynamic SAW potential that travel at the sound velocity up the potential hill of the QPC. The occupancy of a specific dot is determined by the Coulomb interaction between the electron population. Thus, an integer number $n$ of electrons is transported per SAW cycle across the constriction.

Different aspects of this model were later discussed in a number of experimental ${ }^{9|10| 11|12| 13|14| 15}$ and theoretical ${ }^{19|20| 21 \mid 22}$ studies. Deviations from perfectly flat plateaus in the SAW-driven acoustoelectric (AE) current were 


\section{Single-electron transport driven by surface acoustic waves}

attributed to electron tunneling or thermal activation, either intc ${ }^{20}$ or out of 21 the moving quantum dot. Recently, Fletcher et al. suggested an alternative mechanism involving a static quantum dot, either impurity-induced 23 or fabricated on purpose. ${ }^{24}$ The potential barriers of such a static dot are tuned like a turnstile by the low-power SAW.

This short overview already indicates that different effects could be involved in the SAW-driven single electron transport, and that essential transport mechanisms have not been clearly identified yet. Different mechanisms might dominate different samples. Neither can we rule out possible transitions between different mechanisms in the same device.

Cunningham et al. ${ }^{1011}$ demonstrated that the precision of the AE current quantization can be improved by using shallow-etched QPCs. However, till today the absolute accuracy of the AE current is not sufficient for practical applications as a metrological standard! ${ }^{9|10| 11|12| 13|14| 15]}$ Current plateaus, as a function of gate voltage $V_{g}$ or SAW frequency $f$, are not really flat, leading to a rather arbitrary selection of their exact value. Even at nearly optimum conditions, the $\mathrm{AE}$ current at such plateaus is smaller than the ideally expected multiples of $e f$. This reduction as well as the finite slope of the plateaus could result from the enhanced temperature of the 2DEG due to the applied rf power. On the other hand, this could also result from the electron tunneling through the walls of the moving quantum dots which always have a finite thickness and depth. These topics might distract attention from others which are less obvious. Here, we discuss some of the more basic properties or our SAW devices, their response to the SAW frequency (phase) and the gate voltage.

\section{EXPERIMENTAL DETAILS}

Figure 1 shows the layout of our devices: Two aluminum interdigital transducers (IDTs) could be used to generate the SAW. They were deposited $2.6 \mathrm{~mm}$ apart, on both sides of a 2DEG mesa with a QPC in the center. The IDT electrode spacing set the fundamental acoustic wavelength of the transducers to about $1.15 \mu \mathrm{m}$ and their center frequency to around $2.45 \mathrm{GHz}$. The GaAs/AlGaAs heterostructure had a mobility of $105 \mathrm{~m}^{2} /(\mathrm{Vs})$ and a carrier density of $2.8 \cdot 10^{15} \mathrm{~m}^{-2}$, measured in the dark at $10 \mathrm{~K}$. The QPC was patterned by electron-beam lithography. Two semicircular shallow-etched trenches formed a smooth constriction between the two electron reservoirs, whereas the large areas of the 2DEG across the channel served as side gates. The trenches had a curvature radius of $5.0,7.5$ or $10.0 \mu \mathrm{m}$. They were $200 \mathrm{~nm}$ wide and $40 \mathrm{~nm}$ deep. 

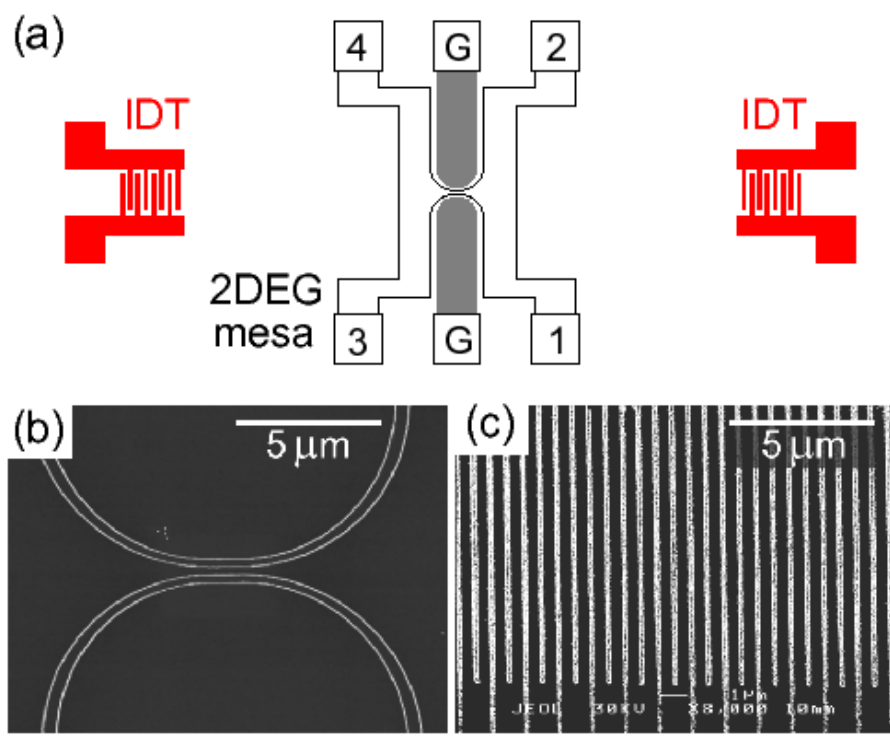

Fig. 1. (Color on-line) (a) Schematic diagram of sample layout: A quantum point contact (QPC) is defined in the 2DEG mesa using a shallow-etch technique. Four Ohmic contacts (1-4) provide electrical connection to the electron reservoirs on both sides of the constriction. The shaded regions are the reservoirs which serve as side gates $(G)$. Two interdigital transducers (IDTs) are deposited on opposite sides of the mesa. Scanning-electron micrographs show (b) the QPC and (c) part of the IDT. The two pairs of bright lines in (b) mark the edges of the shallow-etched trenches that separate the side gates (top and bottom) from the 2DEG (left to right). The parallel white lines in (c) are the IDT fingers. 


\section{Single-electron transport driven by surface acoustic waves}

The rf excitation of power $P$, from an Agilent 8648D or a HewlettPackard HP8673B microwave generator, could be applied to one of the two IDTs, or split up and simultaneously fed to both transducers. With a phase shifter and an attenuator in one of the rf lines, the relative magnitude and the relative phase of both signals could then be varied and adjusted. A low-noise current preamplifier detected the acoustoelectric current.

The samples were investigated either in a ${ }^{3} \mathrm{He}$ refrigerator operating at $1.2 \mathrm{~K}$ or a ${ }^{4} \mathrm{He}$ refrigerator operating at $1.8 \mathrm{~K}$. The lower base temperature of the ${ }^{3} \mathrm{He}$ cryostat did not help to improve the results. In both systems, the 2DEG of our devices was heated up to around $5 \mathrm{~K}$ at the typically applied rf powers of around $10-15 \mathrm{dBm} \cdot \frac{25}{2}$

\subsection{Finding the quantized steps}

Finding the AE plateaus in the $I\left(V_{g}\right)$ characteristics was not always an easy task due to a large number of parameters that had to be tuned: the gate and bias voltage, the SAW power and frequency. For the double-beam configuration, this also included the phase and the relative magnitude of the two counter-propagating SAW beams. Usually, a large number of scans within this parameter space was necessary to find quantized steps in the AE current or to discard the device.

The main components of the device, the IDTs and the QPC, were first characterized by measuring the transmittance of the SAW delay line and the conductance $G$ of the QPC. The $G\left(V_{g}\right)$ characteristics provided information on the pinch-off gate voltage for conductance, whereas the transmittance indicated the pass band at which the SAW transducers should be operated.

The typical optimization procedure was then as follows. The gate voltage on the QPC was set below the conductance pinch-off (typically $\sim 100 \mathrm{mV}$ below) and an rf power of around 10-15 dBm was applied to one of the IDTs. The SAW frequency was scanned to determine the efficiency of each transducer in terms of the AE current carried across the QPC. Figure 2 shows typical $I(f)$ curves of that type. Note the periodic $1.1 \mathrm{MHz}$ oscillations resulting from the SAW reflections from the second, unconnected IDT $!^{2]}$ Those oscillations, characteristic for all our devices, are discussed in the following sections, along with their finer features like the two shifted $1.1 \mathrm{MHz}$ beats clearly visible in Fig. 2(c).

The $I(f)$ traces were inspected for any indications of quantized steps, a flattening of $I(f)$ near integer multiples of $e f$. Intervals of roughly $1 \mathrm{MHz}$ were thus selected around current peaks with such anomalies. The $I\left(V_{g}\right)$ characteristics, similar to those in Fig. 3, were then recorded at fixed fre- 
P. Utko et al.
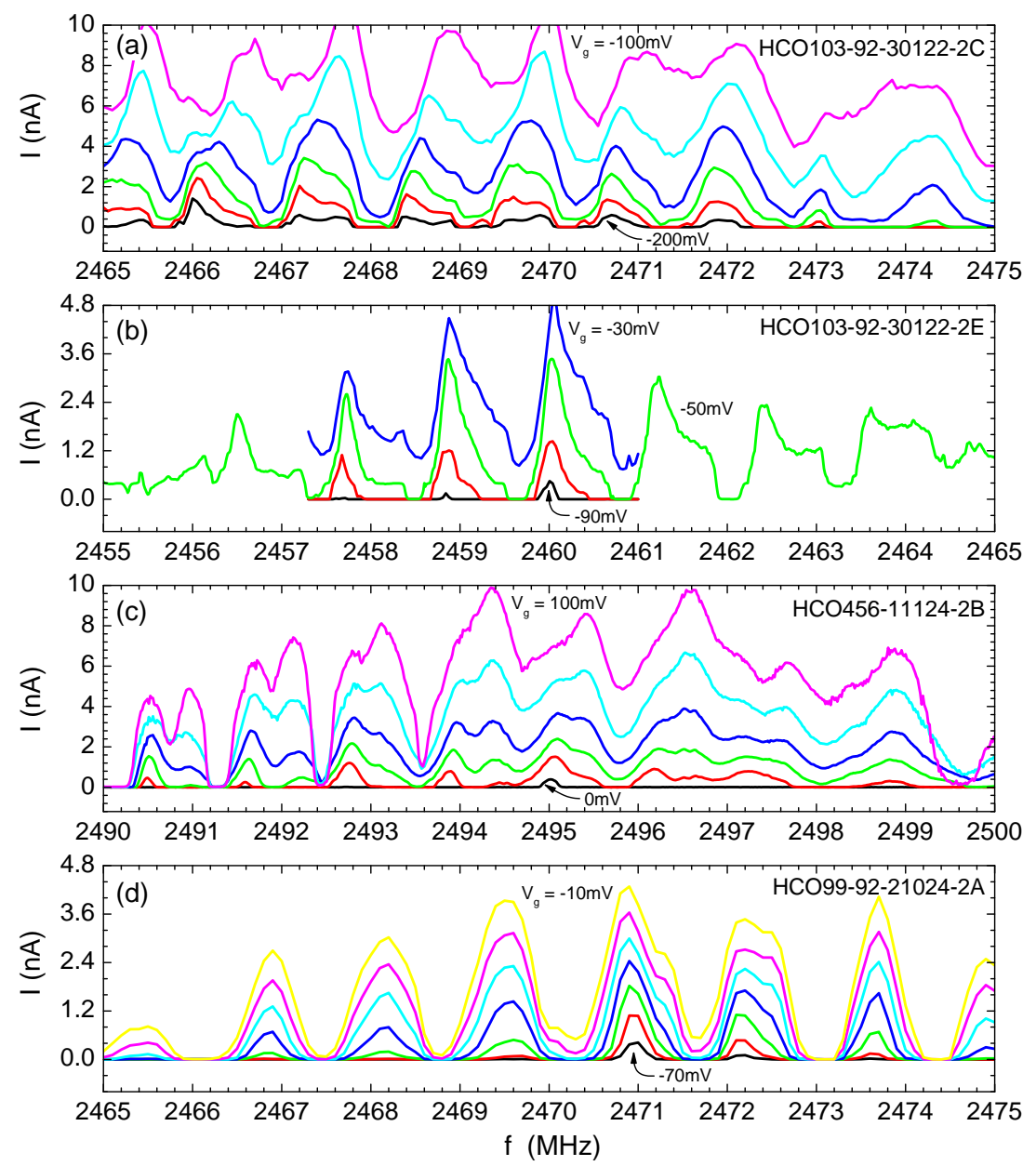

Fig. 2. (Color on-line) Acoustoelectric current $I$ versus SAW frequency $f$ in the indicated range of gate voltages $V_{g}$ below conductance pinch-off. The measurements were performed at $T=1.8 \mathrm{~K}$ for four different samples: (a) HCO103-92-30122-2C, (b) HCO103-92-30122-2E, (c) HCO456-11124-2B, and (d) HCO99-92-21024-2A. 


\section{Single-electron transport driven by surface acoustic waves}
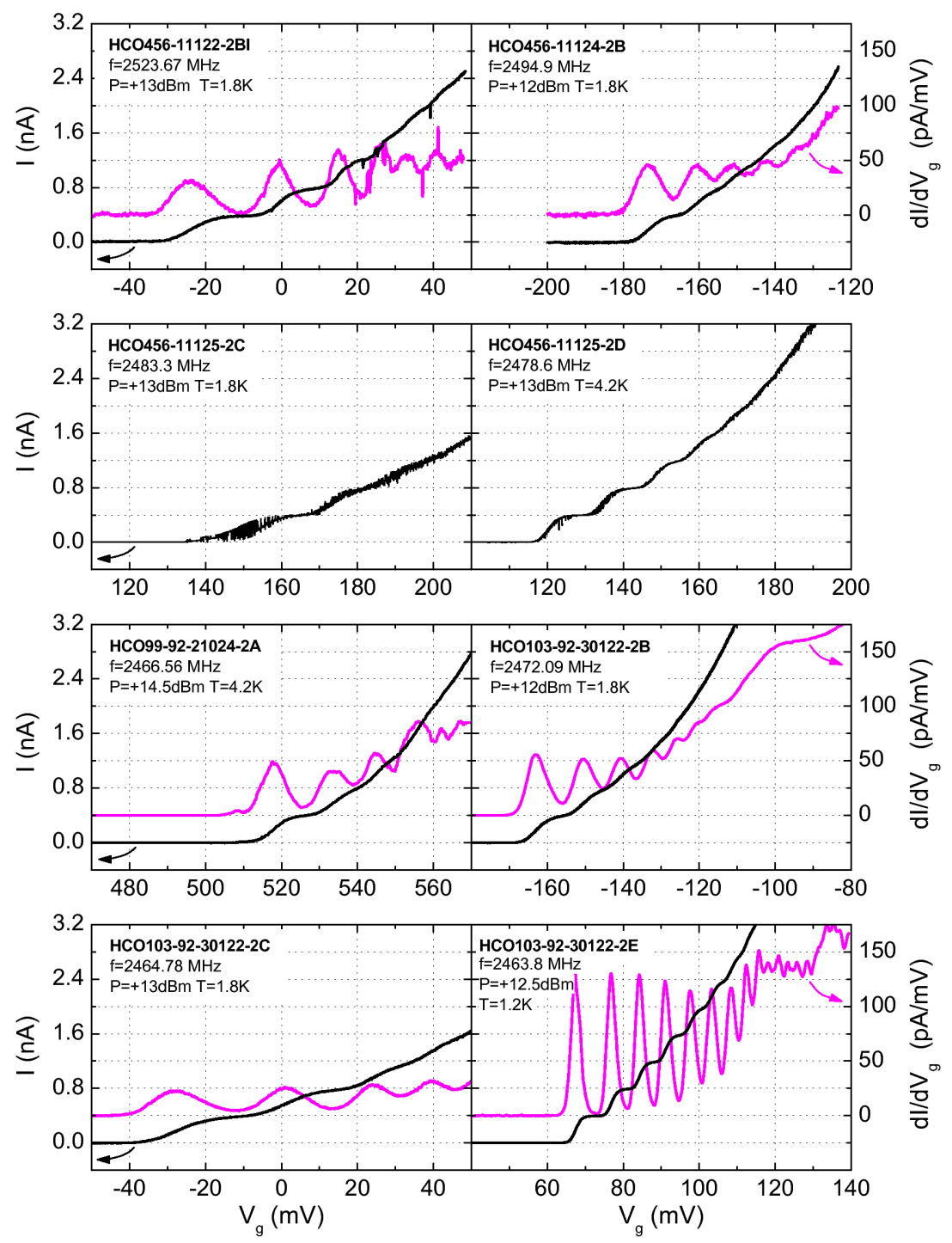

Fig. 3. (Color on-line) (dark) Acoustoelectric current $I$ and (light) transconductance $d I / d V_{g}$ as a function of gate voltage $V_{g}$ for all eight working SAW devices. Frequency $f$, rf power $P$, as well as the base temperature $T$ of the refrigerator are indicated in the consecutive panels. In some of the curves, pronounced random telegraph noise is present. 


\section{P. Utko et al.}

quencies from those ranges, in steps of $\Delta f \approx 0.1 \mathrm{MHz}$. To enhance the resolution of the current plateaus, we monitored as well the transconductance $d I / d V_{g}$. This was done by adding a small $117 \mathrm{~Hz}$ modulation of $d V_{g} \approx 0.5 \mathrm{mV}$ to the gate voltage, and measuring the resulting ac component $d I$ of the acoustoelectric current with a lock-in amplifier. With those precise $I\left(V_{g}, f\right)$ and $d I\left(V_{g}, f\right) / d V_{g}$ maps, the AE transitions could be localized, and extensive scans over the interesting parameter range could be initiated.

\subsection{Counter-propagating SAW beams}

The acoustoelectric current carried across the QPC relies on the dynamic modulation of the electrostatic potential imposed by the SAW. This modulation can be seriously affected in the presence of other spurious signals. For example, the main SAW beam could interfere with an additional counter-propagating beam, which results from the SAW reflections from the second (unconnected) transducer.226 In fact, we consider such as scenario as the most likely explanation for strong current oscillations with respect to frequency, typical for all our devices (Fig. 2).

Two counter-propagating SAW beams can be described as a superposition of a traveling and a (usually weaker) standing wave. The position of the standing wave nodes and antinodes with respect to the QPC is then determined by the phase difference between the two beams. Assuming that the backward beam originates from the SAW reflections from the second transducer, the phase shift depends on a double distance $2 L$ between the point contact and the unconnected IDT. In our structures $2 L \approx 2.6 \mathrm{~mm}$ which should result in a frequency modulation of $\Delta f=v_{S A W} / 2 L \approx 1.1 \mathrm{MHz}$, remarkably close to the beat period observed in our measurements. Here, $v_{S A W} \approx 2800 \mathrm{~m} / \mathrm{s}$ is the velocity of the surface wave. In a previous study by Talyanskii et al., 2 a larger separation between the QPC and the second transducer $2 L \approx 4 \mathrm{~mm}$ yielded a correspondingly smaller beat period of $\Delta f \approx 0.7 \mathrm{MHz}$. In addition, no current oscillations were observed there for a sample with only one IDT.

We note that the SAW potential might also interfere (cross-talk) with the electromagnetic wave irradiated by the transducer. Since the airborne signal reaches the QPC almost instantaneously, the resulting frequency modulation in our devices should then be around $\Delta f=v_{S A W} / L \approx 2.2 \mathrm{MHz}$, unlike the typically observed $1.1 \mathrm{MHz}$ beat.

The second, counter-propagating SAW beam can also be generated on purpose by applying a microwave signal to both transducers. ${ }^{9114}$ This allows a direct control over the relative magnitude and the relative phase between 


\section{Single-electron transport driven by surface acoustic waves}

the two beams. Experiments on the SAW-driven single-electron pumps in a double-beam configuration revealed notable changes in the $I\left(V_{g}\right)$ character-

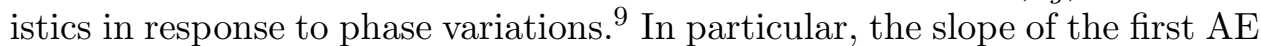
plateau in $I\left(V_{g}\right)$ was significantly reduced for some phase settings. This already indicated that the second SAW beam could be used to further improve the accuracy of the AE current quantization, if only the phase difference between the two counter-propagating beams was properly optimized.914

We emphasize the fact that two facing transducers were deposited on opposite sides of the QPC for all our samples, even though a single IDT is sufficient for the SAW pump operation. This was done in order to increase the yield of working devices and to enable experiments in a double-beam configuration. However, this also implied that no direct comparison was possible with devices comprising only one transducer for which the backward beam should be effectively dampened.

\section{EFFECT OF THE SAW FREQUENCY}

Our measurements of the AE current $I$ and the transconductance $d I / d V_{g}$, versus both the gate voltage $V_{g}$ and the SAW frequency $f$, clearly show the current modulation with respect to frequency, with a period of about 1.1 MHz. However, within this period two frequency intervals can be distinguished for which apparently different sets of AE plateaus dominate the $I\left(V_{g}\right)$ characteristics. This feature shows up whether the SAW is generated by one IDT only (single-beam configuration) or by two facing IDTs (doublebeam configuration). We point out that corresponding intervals can also be resolved when the relative phase between the two counter-propagating SAW beams is varied instead of frequency. Two of our devices are studied in detail: 2C (= HCO103-92-30122-2C) and 2E (= HCO103-92-30122-2E).

\subsection{Single-beam configuration}

Figure 4] shows the acoustoelectric current with respect to both the gate voltage and the SAW frequency for sample 2C. During the measurement, only one IDT was used to generate the SAW (single-beam configuration). The AE plateaus could be resolved for all frequencies within the shown $6 \mathrm{MHz}$ interval. However, their slope as well as their extension along the gate-voltage axis responded very sensitively to variations in frequency.

The AE transitions are easier to resolve in the current derivative. Figure 5 shows a gray-scale plot of the transconductance $d I / d V_{g}$, revealing a periodic structure with respect to frequency with a beat period of roughly 
P. Utko et al.

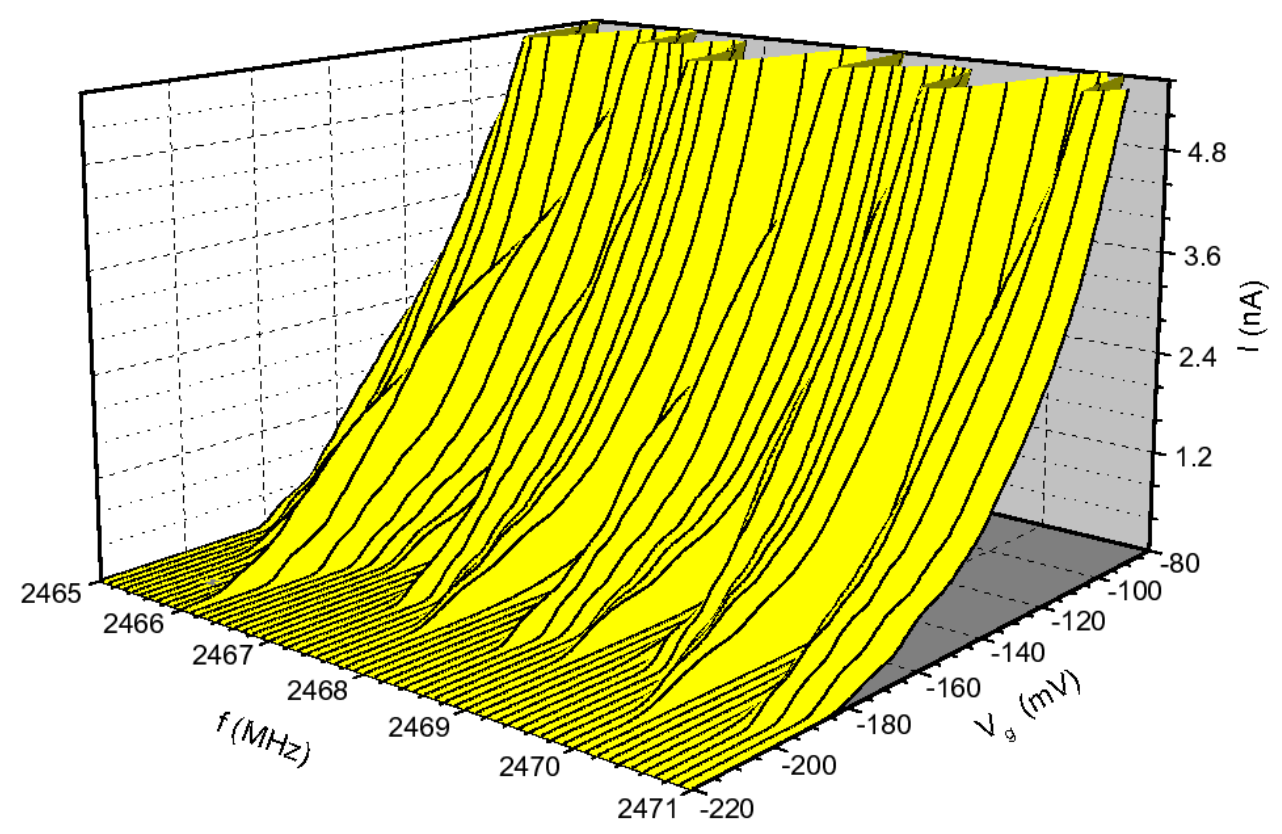

Fig. 4. (Color on-line) AE current $I$ versus gate voltage $V_{g}$ and SAW frequency $f$. The measurement was performed in a single-beam configuration. Device $2 \mathrm{C}$ at $P=+9.8 \mathrm{dBm}$ and $T=1.8 \mathrm{~K}$.

1.1 MHz. However, transconductance minima corresponding to current plateaus do not evolve smoothly over the entire $1.1 \mathrm{MHz}$ interval. On increasing the SAW frequency, broad plateaus in $I\left(V_{g}\right)$ are abruptly replaced by denselypacked quantized steps. Frequencies around which such transitions occur are marked with bars. Arrows indicate another type of transition where the AE current drastically shifts its onset along the $V_{g}$-axis in response to a small change in frequency. This displacement can be as large as $\Delta V_{g} \approx 50 \mathrm{mV}$ over $\Delta f \approx 0.2 \mathrm{MHz}$, as for example around $f \approx 2467.8 \mathrm{MHz}$.

Those features become more apparent in Fig. 6] which shows the selected $I\left(V_{g}\right)$ and $d I\left(V_{g}\right) / d V_{g}$ characteristics from Figs. 4 and 5, respectively. In the following, we describe the frequency evolution of those curves in more detail.

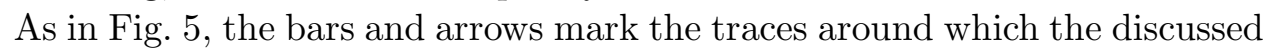
transitions occur.

(i) When the SAW frequency is incremented from $2465.7 \mathrm{MHz}$, the first plateau in $I\left(V_{g}\right)$ broadens with respect to the gate voltage. At the same time, the current onset shifts towards smaller values of $V_{g}$. At $f=2465.9 \mathrm{MHz}$, the first quantized step can no longer be resolved in either the AE current or the transconductance.

(ii) When the frequency is increased above $2465.9 \mathrm{MHz}$, the AE plateaus 


\section{Single-electron transport driven by surface acoustic waves}

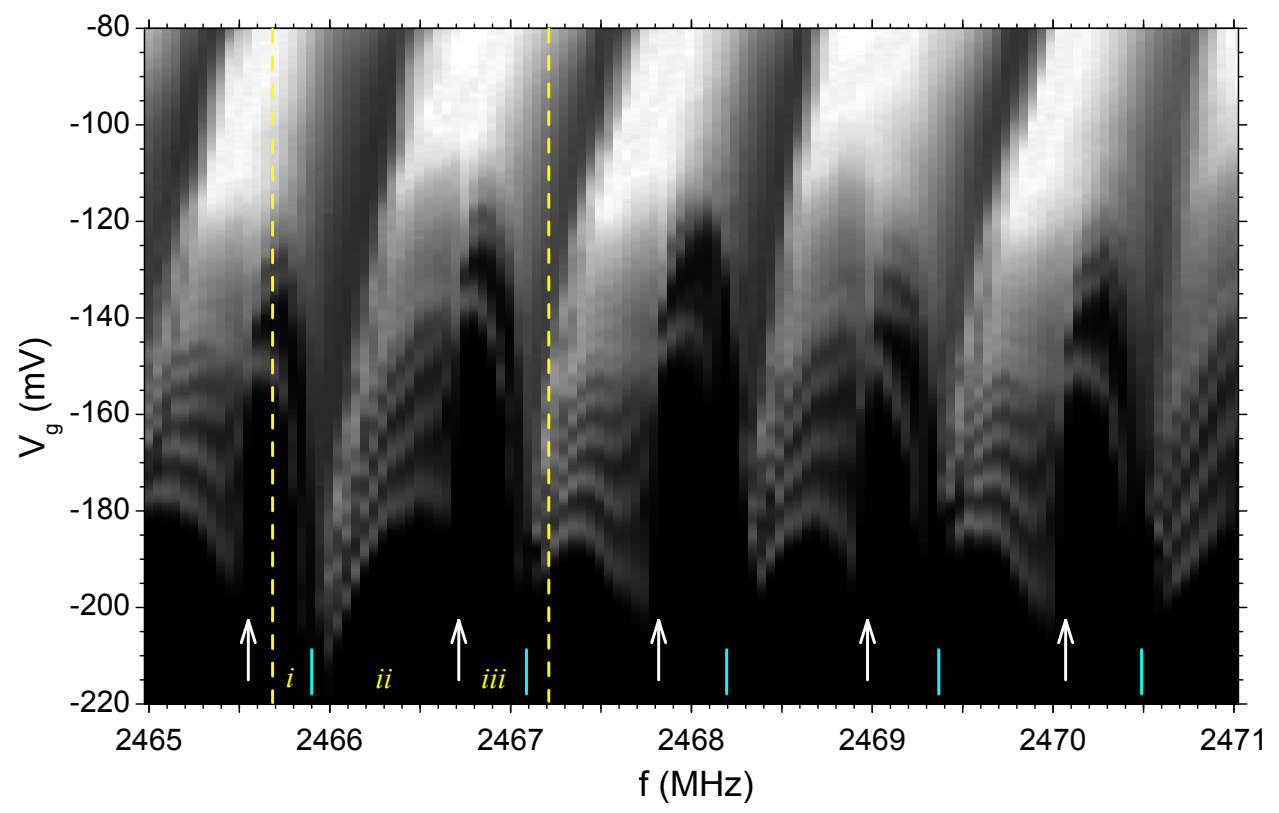

Fig. 5. (Color on-line) Gray-scale coded plot of transconductance $d I / d V_{g}$ with respect to SAW frequency $f$ and gate voltage $V_{g}$. Dark (light) indicates small (large) values of the current derivative. Note the periodic structure with a modulation period of about $1.1 \mathrm{MHz}$. In addition, two smaller intervals can be distinguished within such a period where different sets of AE plateaus seem to prevail. The arrows and bars mark the frequencies around which one set is replaced by another one, see text for details. The dashed lines indicate the frequency range from which the traces in Fig. 6 were selected. Device $2 \mathrm{C}$ at $P=+9.8 \mathrm{dBm}$ and $T=1.8 \mathrm{~K}$. 
P. Utko et al.

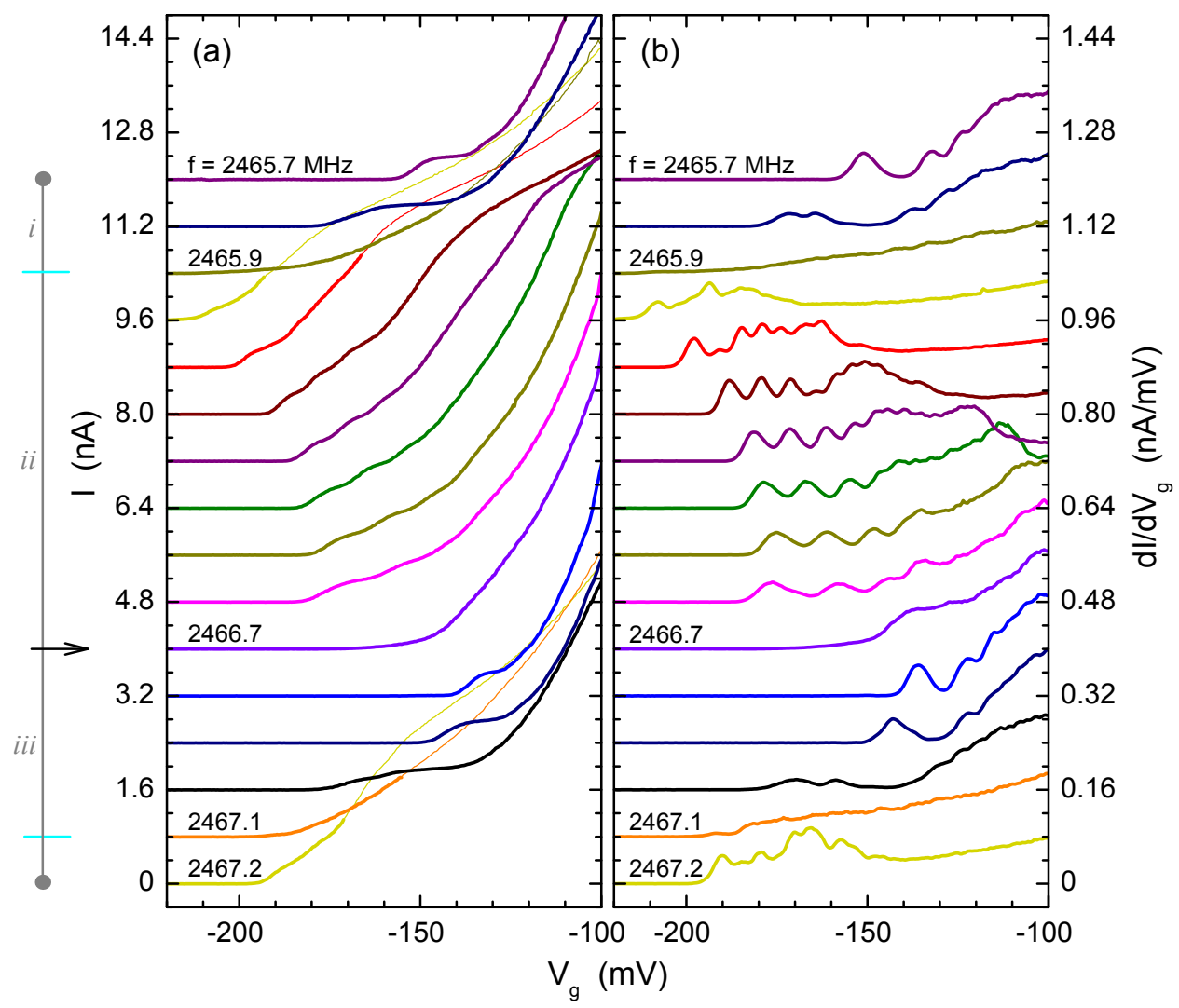

Fig. 6. (Color on-line) (a) AE current $I$ and (b) transconductance $d I / d V_{g}$ as a function of gate voltage $V_{g}$. The 16 current (transconductance) traces were recorded at fixed SAW frequencies from 2465.7 to $2467.2 \mathrm{MHz}$, in steps of $0.1 \mathrm{MHz}$. The curves were successively offset in the vertical direction by $0.8 \mathrm{nA}(0.08 \mathrm{nA} / \mathrm{mV})$. Device $2 \mathrm{C}$ at $P=+9.8 \mathrm{dBm}$, and $T=1.8 \mathrm{~K}$. 


\section{Single-electron transport driven by surface acoustic waves}

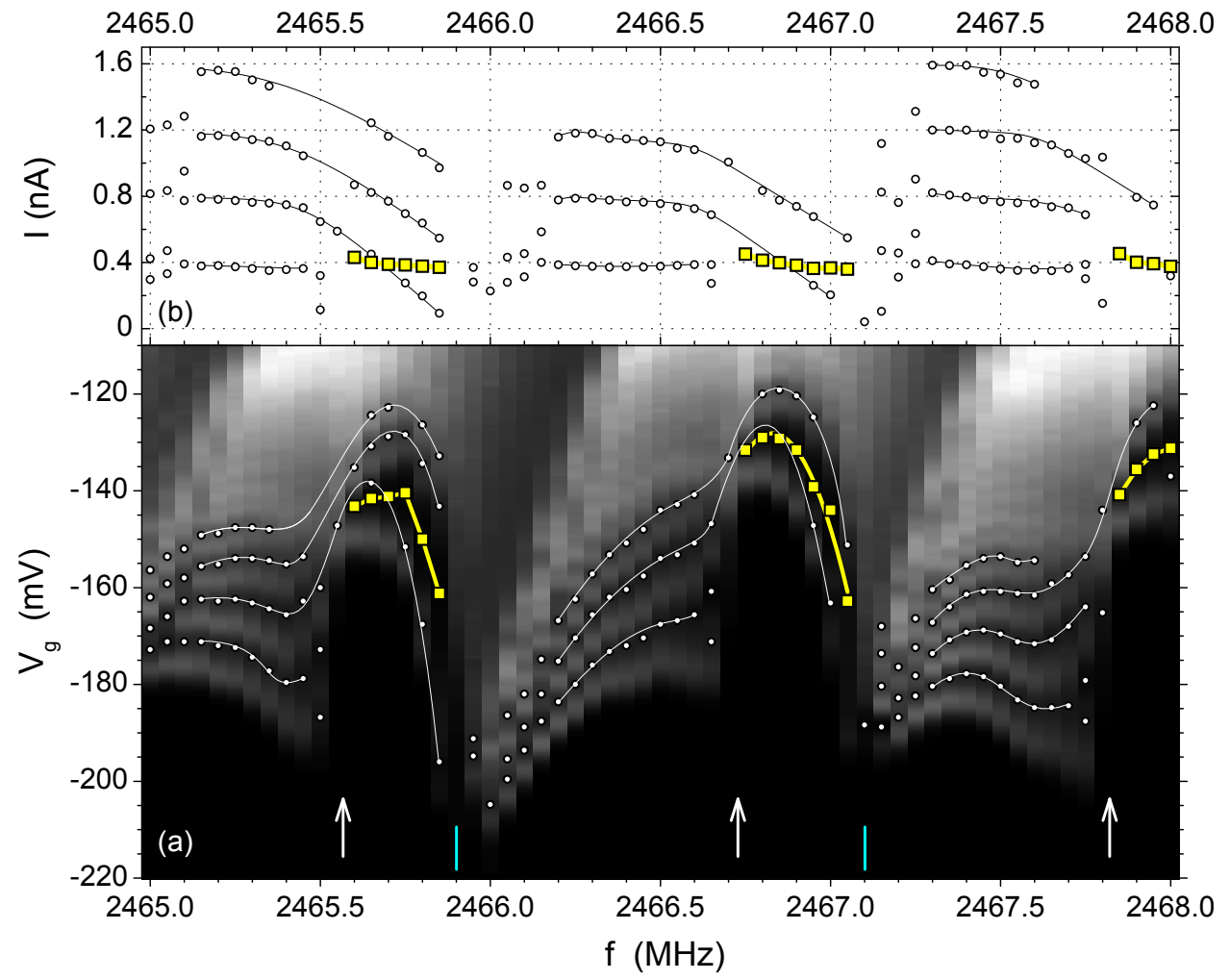

Fig. 7. (Color on-line) (a) Gray-scale plot of transconductance $d I / d V_{g}$ with respect to SAW frequency $f$ and gate voltage $V_{g}$. Dark (light) indicates small (large) values of the derivative. Open circles and full squares mark the position of transconductance minima. (b) AE current $I$ at the minima indicated in (a). In both (a) and (b), two different kinds of AE plateaus can be distinguished. Solid lines through the data points are guides to the eye. Device $2 \mathrm{C}$ at $P=+9.8 \mathrm{dBm}$ and $T=1.8 \mathrm{~K}$. 


\section{P. Utko et al.}

reappear in the $I\left(V_{g}\right)$ traces. However, they are much less pronounced than those in $(i)$, as both their slope and number per gate-voltage interval has increased. This new set of plateaus evolves smoothly until $f \approx 2466.7 \mathrm{MHz}$, where the onset of the $I\left(V_{g}\right)$ characteristics abruptly shifts towards higher gate voltages. For the traces obtained at $f=2466.6$ and $2466.8 \mathrm{MHz}$, this displacement is as large as $\Delta V_{g} \approx 40 \mathrm{mV}$.

(iii) Above $2466.7 \mathrm{MHz}$, another set of quantized steps develops in $I\left(V_{g}\right)$. It continues until $f=2467.1 \mathrm{MHz}$, behaving in a similar way as the corresponding set in $(i)$.

In both Figs. 5 and 6. two frequency intervals can be distinguished within a beat period of $\sim 1.1 \mathrm{MHz}$ where two different sets of AE plateaus dominate the $I\left(V_{g}\right)$ characteristics. Those two sets seem to replace each other around certain frequencies, indicated by the arrows and bars. However, in some cases, both sets can also appear simultaneously in the $I\left(V_{g}\right)$ traces, as if they were superposed onto each other. The dominating set consists then of broad current plateaus that are well-defined at the expected multiples of $e f$. On the other hand, weakly-pronounced steps belonging to the second set are formed below those ideal values, as shown in Fig. 7 .

Both sets respond differently to the SAW frequency as becomes apparent, for example, in the following range $f=2465.7-2465.9 \mathrm{MHz}$ in Fig. 7 When the frequency is incremented within such interval, one plateau in $I\left(V_{g}\right)$ remains close to the expected value of $e f \approx 400 \mathrm{pA}$, while the other set of quantized steps appears at lower and lower currents. The plateau at $I=e f$ is broad and well-pronounced with respect to the gate voltage, while the steps away from the expected multiples of ef are barely indicated in the $I\left(V_{g}\right)$ characteristics.

\subsection{Double-beam configuration}

Two separate sets of current plateaus, appearing within the $1.1 \mathrm{MHz}$ beat period, could also be observed when the device was operated in a double-beam configuration.

Figures 8 and 9 show the acoustoelectric current and the transconductance, respectively, for device 2E. During the measurement, two counterpropagating SAW beams were generated on purpose from the two transducers on opposite sides of the QPC. An rf power of $P=13.5 \mathrm{dBm}$ was applied to the driving IDT, while the excitation applied to the second transducer was attenuated by $8 \mathrm{~dB}$. In order to obtain the flattest plateaus in the acoustoelectric current, the relative phase between the two counter-propagating SAW beams was optimized. The slope of the first plateau was thus reduced 


\section{Single-electron transport driven by surface acoustic waves}

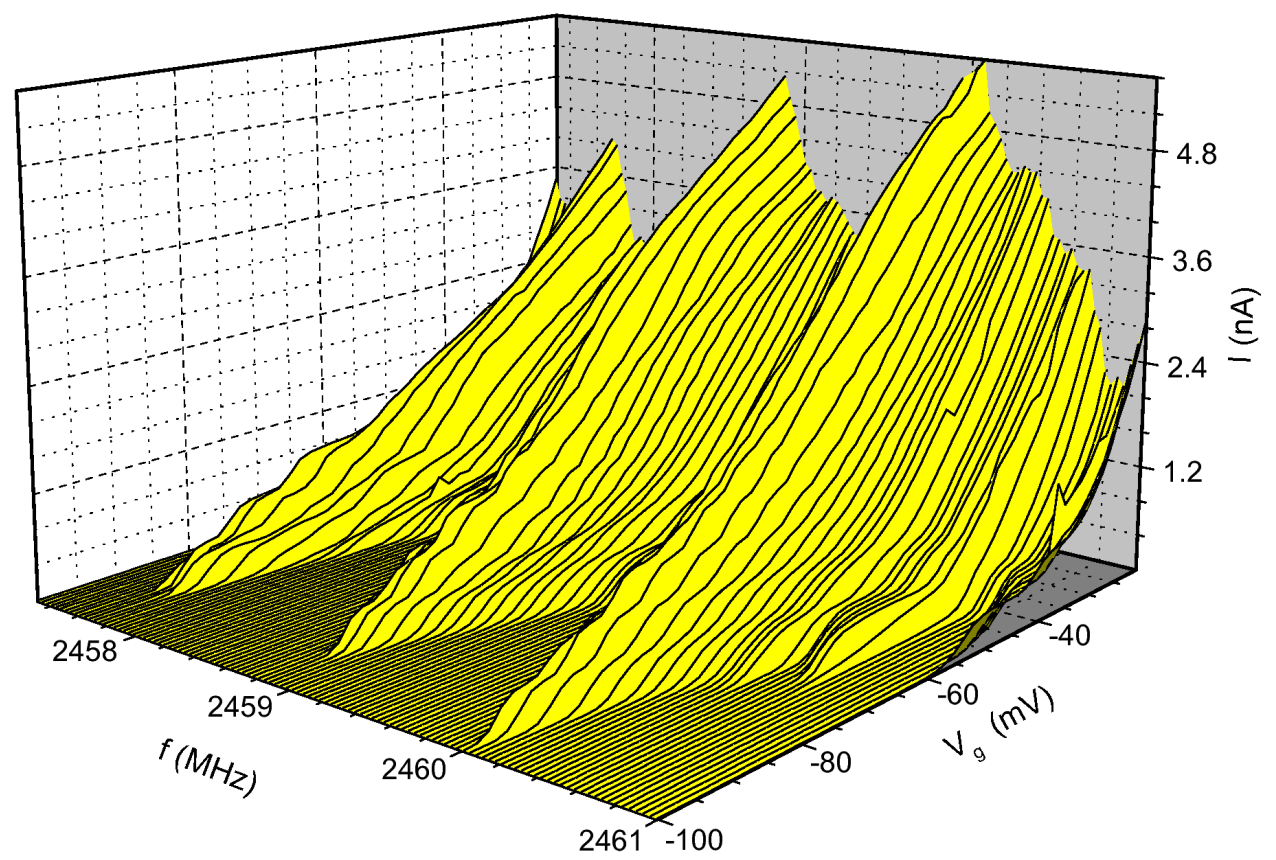

Fig. 8. (Color on-line) AE current $I$ versus gate voltage $V_{g}$ and SAW frequency $f$. The measurement was performed in a double-beam configuration. The microwave signal of $P=+13.5 \mathrm{dBm}$ was applied to the driving transducer, while the excitation applied to the second IDT was attenuated by $8 \mathrm{~dB}$. Device $2 \mathrm{E}$ at $T=1.2 \mathrm{~K}$.

by an order of magnitude with respect to the case when the second beam was off. 14

As for the single-beam configuration, both the AE current (Fig. 8) and the transconductance (Fig. 9) reveal a very clear $1.1 \mathrm{MHz}$-beating. Two frequency intervals can be distinguished within this period, where two different sets of the AE plateaus dominate the $I\left(V_{g}\right)$ characteristics.

Figure 10 shows the selected $I\left(V_{g}\right)$ and $d I\left(V_{g}\right) / d V_{g}$ traces from Figs. 8 and 9 , respectively. Consecutive curves were taken at incremented frequencies, in steps of $0.1 \mathrm{MHz}$. Note that the frequency response of those curves is very similar to the one described in the previous section:

(i) When the frequency is increased from $f=2458.6 \mathrm{MHz}$, the onset of the acoustoelectric current shifts towards lower gate voltages. At the same time, the AE plateaus broaden with respect to $V_{g}$. This trend continues until $f=2458.8 \mathrm{MHz}$.

(ii) Around $f=2458.8 \mathrm{MHz}$, a new set of densely-spaced plateaus develops near the onset of $I\left(V_{g}\right)$. This new set evolves smoothly until 
P. Utko et al.

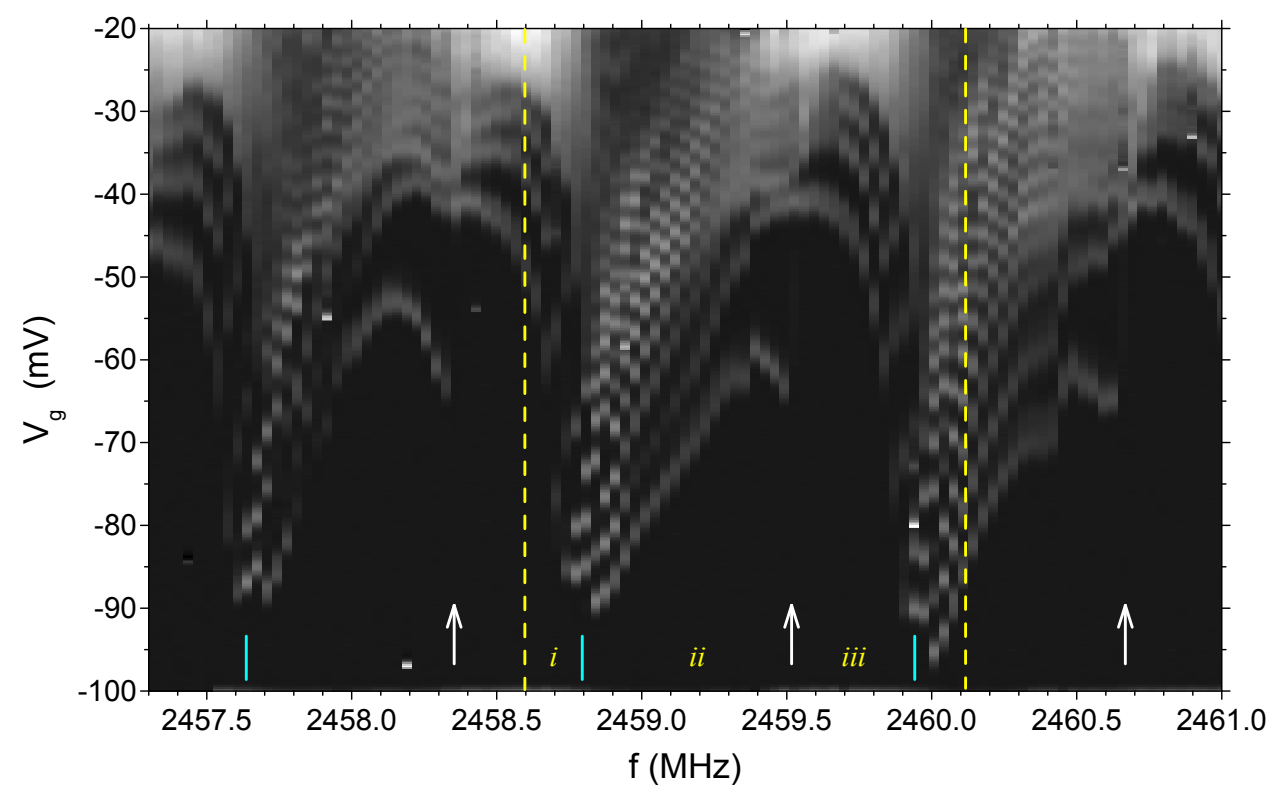

Fig. 9. (Color on-line) Gray-scale plot of transconductance $d I / d V_{g}$ with respect to SAW frequency $f$ and gate voltage $V_{g}$. Dark (light) indicates small (large) current derivative. The measurement was performed in a doublebeam configuration. Bars and arrows indicate frequencies around which one set of AE plateaus is replaced by another. The dashed lines indicate the frequency range from which the traces in Fig. 10 were selected. Device 2E at $T=1.2 \mathrm{~K}$. 
Single-electron transport driven by surface acoustic waves

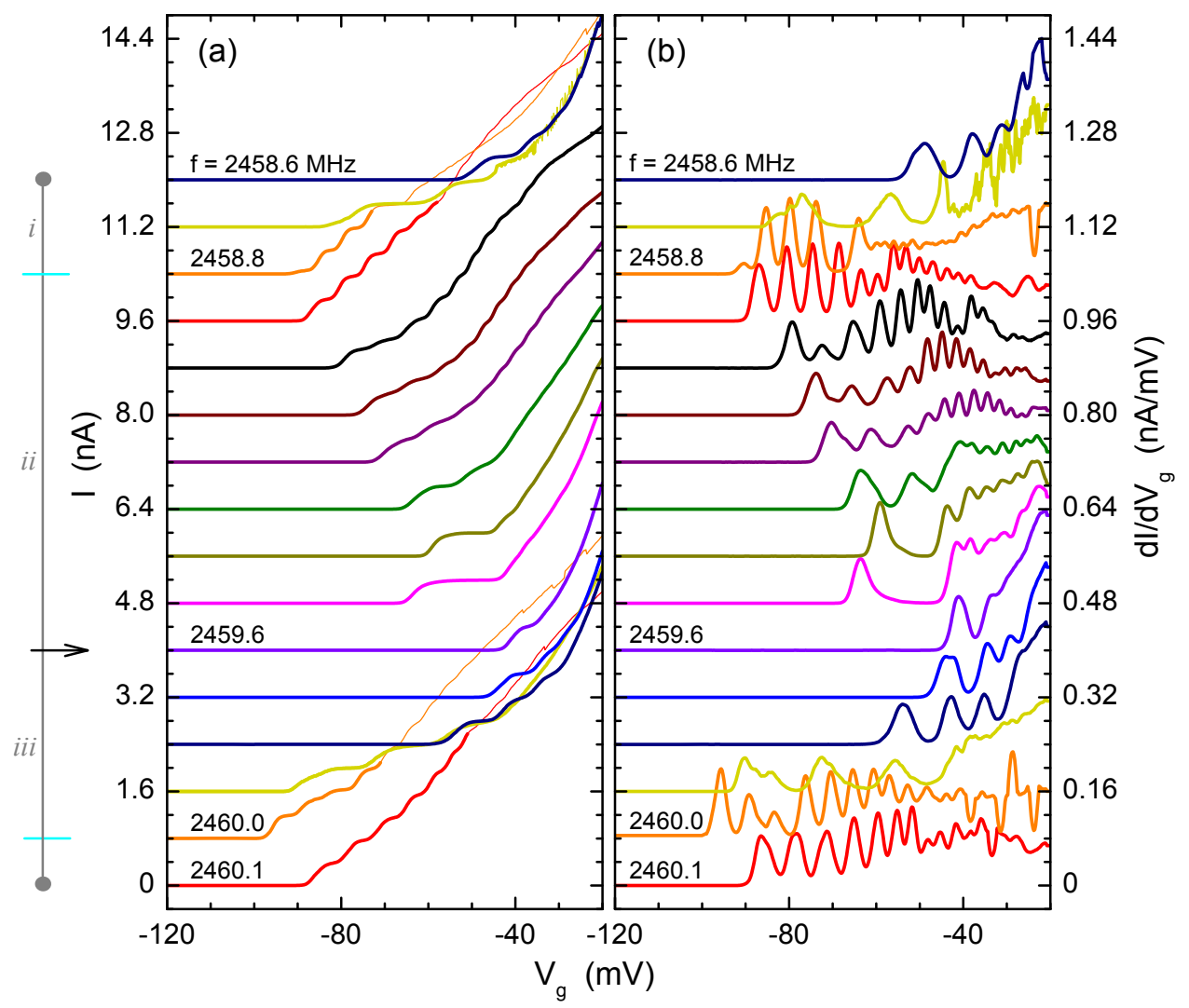

Fig. 10. (Color on-line) (a) AE current $I$ and (b) transconductance $d I / d V_{g}$ as a function of gate voltage $V_{g}$. The 16 current (transconductance) traces were recorded at fixed SAW frequencies from 2458.6 to $2460.1 \mathrm{MHz}$, in steps of $0.1 \mathrm{MHz}$. The curves were successively offset in the vertical direction by $0.8 \mathrm{nA}(0.08 \mathrm{nA} / \mathrm{mV})$. Device $2 \mathrm{E}$ at $T=1.2 \mathrm{~K}$. 


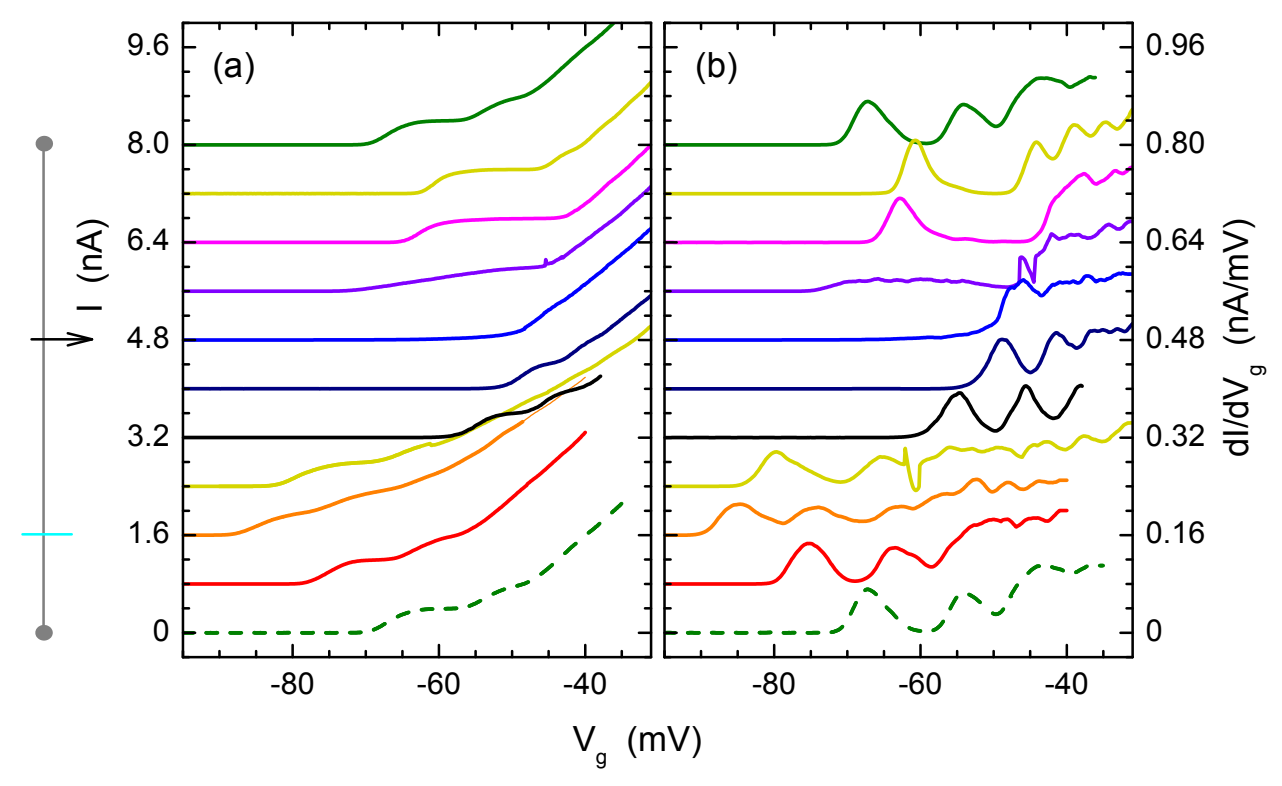

Fig. 11. (Color on-line) (a) Acoustoelectric current $I$ and (b) transconductance $d I / d V_{g}$ as a function of gate voltage $V_{g}$. The consecutive traces were measured at different phase shifts between the main and the counterpropagating SAW beam, in steps of about $18^{\circ}$ (from top to bottom). The dashed curve completes the $2 \pi$ cycle. The traces are vertically offset for clarity. The power applied to the main IDT was $P=+13.5 \mathrm{dBm}$, while the power at the second IDT was attenuated by $8 \mathrm{~dB}$. Device $2 \mathrm{E}$ at $f=2459.4 \mathrm{MHz}$ and $T=1.2 \mathrm{~K}$. 


\section{Single-electron transport driven by surface acoustic waves}

$f \approx 2459.6 \mathrm{MHz}$. Close to this frequency, the current onset abruptly shifts towards larger gate voltages.

(iii) The abrupt shift of the current onset marks the transition to the next set of the AE plateaus, which corresponds to the one in $(i)$.

As for device $2 \mathrm{C}$, for some $\mathrm{SAW}$ frequencies, quantized steps in the acoustoelectric current can be observed both at the expected multiples of $I=$ nef and away from those values, see for example the traces obtained at $f=2459.9$ or $2460.0 \mathrm{MHz}$.

The frequency response of the $I\left(V_{g}\right)$ characteristics (Figs. 6] and 10) closely resembles their response to the relative phase between the two counterpropagating SAW beams (Fig. 11): When the frequency (phase) is varied, two intervals can be distinguished within a period of $1.1 \mathrm{MHz}(2 \pi)$ where two different sets of AE plateaus dominate the $I\left(V_{g}\right)$ characteristics. Around certain frequencies (phases), those two sets of quantized steps seem to replace each other. This might result in abrupt shifts of the current onset in $I\left(V_{g}\right)$, as for the traces indicated by the arrows in Figs. 6] and 10 (Fig. 11]for phase). The other, more subtle, type of transition takes place for the curves marked with the bars.

\subsection{Other beat periods}

The frequency response characteristics of our devices, similar to those in Figs. 4- 10, were stable as long as the device was kept cold. When the sample was thermally cycled to room temperature and cooled down again, the results differed in details from those obtained previously. However, in most cases they remained qualitatively similar to those described above.

Only on few cool-downs, a larger beat period than $1.1 \mathrm{MHz}$ was observed in the $I\left(V_{g}, f\right)$ characteristics. Figure 12(b) shows an example where the dominant beat period has changed to about 2.2 MHz. This might suggest an enhanced role of cross-talk, the interference between the dynamic SAW potential and the airborne electromagnetic wave irradiated by the transducer. The structure of the AE transitions in Fig. 12 is indeed very complex. At present, we cannot provide a generalized description of those results like for the $1.1 \mathrm{MHz}$ oscillations in the preceding sections. Nevertheless, the data in Fig. 12 also show different sets of current plateaus.

\section{DISCUSSION}

The frequency response measurements presented here reveal a complicated pattern of the AE transitions. Their most apparent feature, the 


\section{P. Utko et al.}

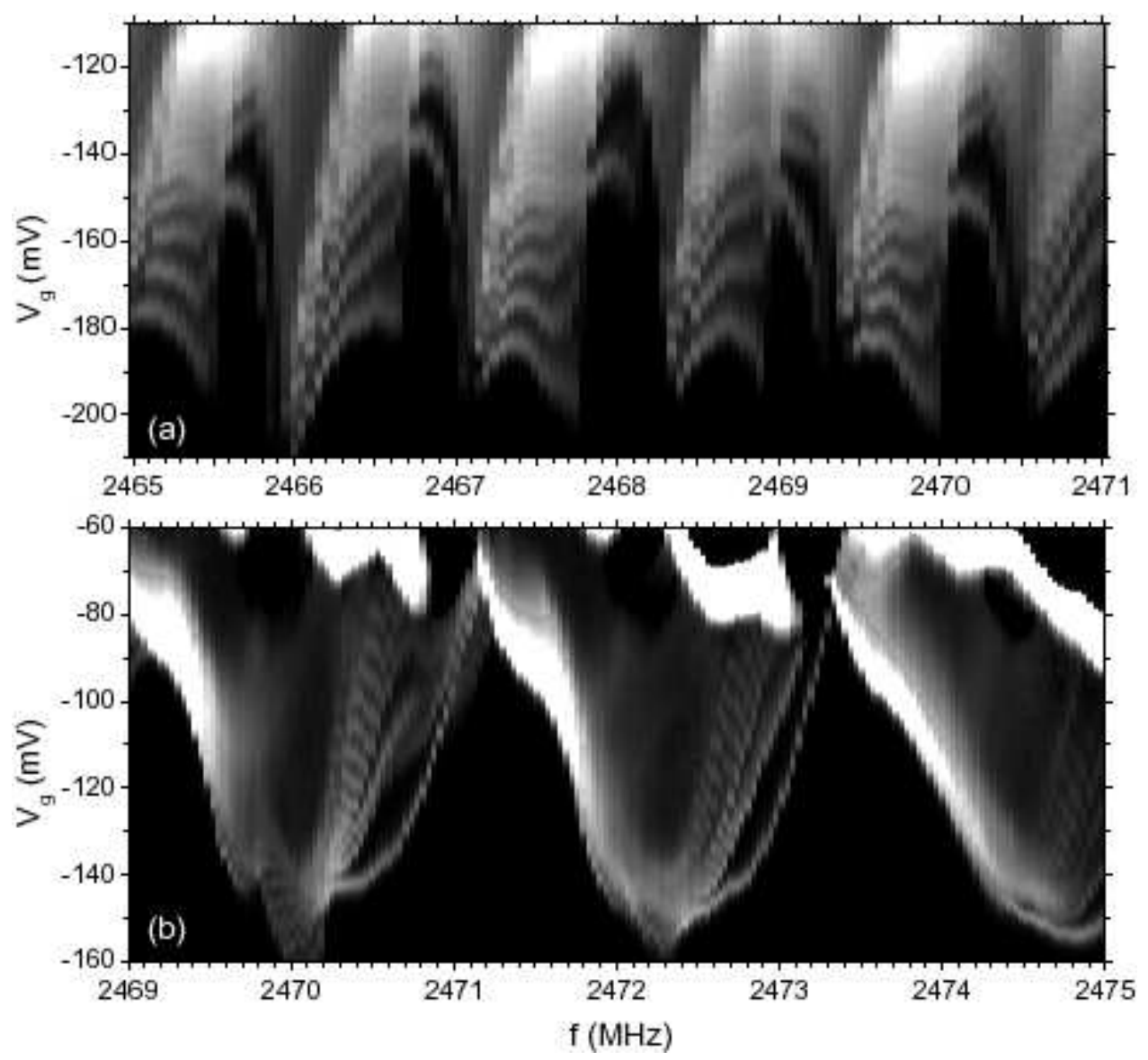

Fig. 12. Transconductance $d I / d V_{g}$ with respect to SAW frequency $f$ and gate voltage $V_{g}$ after two different cool downs from room temperature. Note the change in the period of frequency modulation from (a) $1.1 \mathrm{MHz}$ to (b) $2.2 \mathrm{MHz}$. The microwave power applied to the SAW transducer differed slightly, being (a) $+9.8 \mathrm{dBm}$ and (b) $+10.8 \mathrm{dBm}$. Device $2 \mathrm{C}$ at $T=1.8 \mathrm{~K}$. 


\section{Single-electron transport driven by surface acoustic waves}

1.1 MHz beating of the acoustoelectric current, immediately reminds us of the interference patterns due to a standing wave. In fact, Fig. 11 demonstrates that the results obtained while varying the SAW frequency closely resemble those when the phase is controlled between two counter-propagating SAW beams. The period of $1.1 \mathrm{MHz}$ corresponds to a $2 \pi$ phase shift. This already indicates that the standing wave matters, but it does not specify where to find its nodes.

We have further demonstrated that two sub-intervals can be distinguished within this period where two different sets of AE plateaus dominate the $I\left(V_{g}\right)$ characteristics. At certain frequencies, one set of quantized steps is replaced by the other one. In some cases, they can even appear simultaneously in the $I\left(V_{g}\right)$ traces, as if they were superposed on each other. In such a situation, quantized steps can be resolved in the AE current both near the expected multiples of ef and at lower values, as shown in Fig. 7 . Note that those two sets of AE plateaus respond differently to the SAW frequency.

\subsection{Parallel channels?}

Different kinds of AE plateaus could be due to the presence of parallel channels for the SAW-driven electrons. This could be explained, for example, by branching of the electron flux at the entrance to the QPC. Topinka et al. ${ }^{27 / 28}$ recently demonstrated spatially resolved images of the electron flow in the 2DEG, indicating different current strands at the entrance and the exit of the point contact. This was attributed to focusing of the electron trajectories by a large ensemble of small $\left(\sim 0.1 \epsilon_{F}\right)$ ripples in the background potential, caused by impurities or donors. In our experiments, such branching of the electron flux could affect how the moving quantum dots are populated with electrons. On the other hand, separate SAW channels could also be present in the QPC itself. In the vicinity of a closed point contact, screening of impurity potentials is reduced. Hence, the size of such potential fluctuations could be much larger in the QPC region $(\sim 50 \mathrm{mV})$ than in the 2 DEG $(\sim 2 \mathrm{mV}) .29130$ This, in turn, could lead to the formation of separate pathways through the constriction.

A quantized acoustoelectric current could be obtained separately for each of those discrete trajectories. Note that changing the SAW frequency (or phase) shifts the nodes and antinodes of the standing wave. Therefore, the amplitude of the SAW modulation is affected at a particular position within the QPC. At certain frequencies (phases), different SAW channels could thus be opened or suppressed, leading to abrupt transitions between different sets of quantized steps in the acoustoelectric current. However, 


\section{P. Utko et al.}

such a scheme should be strongly dependent on the exact configuration of random impurity potentials, whereas our results show systematic variations of the AE plateaus. Therefore, we consider such a scenario rather unlikely.

\subsection{Effect of the short barrier(s)}

The SAW-driven single-electron transport is usually described using a model of moving quantum dots, first suggested by Shilton et al. ${ }^{112}$ In such a model, single electrons trapped in the local minima of the dynamic SAW potential are transferred across the QPC barrier, which is assumed to be long with respect to the SAW wavelength. As the dot moves towards the center of the constriction and its size decreases, the Coulomb repulsion between the trapped electrons restricts their number inside the dot, forcing some of them to escape back to the 2DEG reservoir they where captured from. Thus, the minimum size of the dot determines the final number $n$ of transferred electrons. In later theoretical studies, $\frac{1921}{21}$ the role of the electron escape process for the AE current quantization has been investigated in more detail. Another possible quantization mechanism, the electron capture process at the entrance to the constriction, has been considered by Flensberg et al. ${ }^{20}$

In all those models, $\frac{121920121 / 22}{2}$ the static barrier of the QPC is assumed to be long with respect to the SAW wavelength. However, we believe that this is not entirely valid for our devices. In spite of large nominal lengths of our QPCs $(\sim 2 \mu \mathrm{m})$, their properties are determined by rather short barriers of around $0.2 \mu \mathrm{m}, \frac{31}{,}$ that is about $1 / 5$ of the SAW wavelength. Such a short barrier within the QPC channel could reduce the number of electrons that are further carried in a moving quantum dot.

We emphasize the main difference with respect to the models relying solely on long QPC barriers ${ }^{1 / 2|19| 20|21| 22}$ where only the low-energy electrons at the bottom of the dot are transferred across the constriction. This is no longer the case if the moving quantum dot approaches a short (though high) barrier. Electrons in the lowest energy states of the SAW minimum are then held back at the barrier and return to the reservoir they originated from. Only those with higher energy are transferred across the QPC and contribute to the AE current.

Note that expelling of low-energy electrons at the short static barrier can take place in series with the quantization mechanisms described in previous

models, relying on the escape or capture of high-energy electrons. 1 |2|19|20|21|22 The originally quantized current can thus be further reduced at the short barrier, also to a new quantized level. This could explain Fig. 7? The higher order plateaus (thin lines/points) are lowered when the second mechanism 


\section{Single-electron transport driven by surface acoustic waves}

(thick line/points) sets in. Thus, this simple model would provide an explanation to the presence of at least two different kinds of AE current plateaus. It could also explain the transition between the series of broad and wellpronounced AE plateaus starting at low $V_{g}$, and the narrowly-spaced and barely-pronounced quantized steps at larger gate voltages, as observed in the $I\left(V_{g}\right)$ characteristics in Figs. 3, 6, and 10 .

One could further speculate that, at very narrow channels, more than one barrier is present in the constriction. One set of the AE plateaus could then result from operating the device as a static quantum dot with two separate barriers of comparable magnitude, as found by Fletcher et al. . $^{23}$ Whereas a second set could be due to a single barrier. In few cases for some of our devices, we indeed observed that the Coulomb blockade peaks at zero SAW power, indicating the formation of a static quantum dot, evolved into the AE current plateaus when the SAW amplitude was increased $[32$

\section{CONCLUSION}

The quantization of the AE current as a function of the gate voltage and the SAW frequency reveals a rather involved picture with several transitions between distinct transport regimes. Changing the SAW frequency has the same effect as changing the phase of a counter-propagating SAW beam. This indicates the presence of a standing wave, which either enhances or reduces locally the potential profile along the QPC channel. This profile determines the quantization condition for the AE current. Still, the exact position of the standing-wave node, and whether it reduces or increases the electrostatic potential there, remains unknown.

We have distinguished two different regimes of the $\mathrm{AE}$ current flow. A discrete number of electrons can be captured and transported across the QPC in the minima of the SAW potential, as already described in Refs. 1 2 19 20 21 22, The second mechanism results from the short barriers of our QPCs. Their presence ${ }^{31}$ already implies that the conventional picture of a conveyor-belt-like transport across the QPC might not be entirely valid for our devices. The number of electrons transferred per SAW cycle across the short barrier is determined by its height: Only high-energy electrons can pass it in forward direction. Thus, it is possible that an already quantized current can be further changed (reduced) to a new quantized condition. 
P. Utko et al.

\section{ACKNOWLEDGMENTS}

This work was supported by the European Commission FET Project SAWPHOTON. P.U. acknowledges support from EC FP6 funding (contract no. FP6-2004-IST-003673).

\section{REFERENCES}

1. J. M. Shilton, V. I. Talyanskii, M. Pepper, D. A. Ritchie, J. A. Frost, C. J. B. Ford, C. G. Smith, and G. A. C. Jones, J. Phys.: Condens. Matter 8, L531 (1996).

2. V. I. Talyanskii, J. M. Shilton, M. Pepper, C. G. Smith, C. J. B. Ford, E. H. Linfield, D. A. Ritchie, and G. A. C. Jones, Phys. Rev. B 56, 15180 (1997).

3. N. M. Zimmermann and M. W. Keller, Meas. Sci. Technol. 14, 1237 (2003).

4. J. L. Flowers and B. W. Petley, Rep. Prog. Phys. 64, 1191 (2001).

5. L. P. Kouwenhoven, A. T. Johnson, N. C. van der Vaart, C. J. P. M. Harmans, and C. T. Foxon, Phys. Rev. Lett. 67, 1626 (1991).

6. M. W. Keller, A. L. Eichenberger, J. M. Martinis, and N. M. Zimmermann, Science 285, 1706 (1999).

7. M. Switkes, C. M. Markus, K. Campman, and A. C. Gossard, Science 283, 1905 (1999).

8. J. P. Pekola, A. B. Zorin, and M. A. Paalanen, Phys. Rev. B 50, 11255 (1994)

9. J. Cunningham, V. I. Talyanskii, J. M. Shilton, M. Pepper, M. Y. Simmons, and D. A. Ritchie, Phys. Rev. B 60, 4850 (1999).

10. J. Cunningham, V. I. Talyanskii, J. M. Shilton, M. Pepper, A. Kristensen, and P. E. Lindelof, Phys. Rev. B 62, 1564 (2000).

11. J. Cunningham, V. I. Talyanskii, J. M. Shilton, M. Pepper, A. Kristensen, and P. E. Lindelof, J. Low Temp. Phys. 118, 555 (2000).

12. J. Ebbecke, G. Bastian, M. Blcker, K. Pierz, and F. J. Ahlers, Appl. Phys. Lett. 77, 2601 (2000).

13. J. Ebbecke, K. Pierz, F. J. Ahlers, Physica E 12, 466 (2002).

14. P. Utko, K. Gloos, J. Bindslev Hansen, and P. E. Lindelof, Acta Phys. Pol. A 103, 533 (2003).

15. K. Gloos, P. Utko, J. Bindslev Hansen, and P. E. Lindelof, Phys. Rev. B 70, 235345 (2004).

16. C. L. Foden, V. I. Talyanskii, G. J. Milburn, M. L. Leadbeater, and M. Pepper, Phys. Rev. A 62, 011803 (2000).

17. C. H. W. Barnes, J. M. Shilton, and A. M. Robinson, Phys. Rev. B 62, 8410 (2000).

18. S. Furuta, C. H. W. Barnes, and C. J. L. Doran, Phys. Rev. B 70, 205320 (2004).

19. G. R. Aizin, G. Gumbs, M. Pepper, Phys. Rev. B 58, 10589 (1998).

20. K. Flensberg, Q. Niu, M. Pustilnik, Phys. Rev. B 60, R16 291 (1999).

21. A. M. Robinson and C. H. W. Barnes, Phys. Rev. B 63, 165418 (2001). 


\section{Single-electron transport driven by surface acoustic waves}

22. V. Kashcheyevs, A. Aharony, and O. Entin-Wohlman, Eur. Phys. J. B 39, 385 (2004).

23. N. E. Fletcher, J. Ebbecke, T. J. B. M. Janssen, F. J. Ahlers, M. Pepper, H. E. Beere, and D. A. Ritchie, Phys. Rev. B 68, 245310 (2003).

24. J. Ebbecke, N. E. Fletcher, T. J. B. M. Janssen, F. J. Ahlers, M. Pepper, H. E. Beere, and D. A. Ritchie, Appl. Phys. Lett. 84, 4319 (2004).

25. P. Utko, P. E. Lindelof, and K. Gloos, Appl. Phys. Lett. 88, 202113 (2006).

26. S. Datta, Surface acoustic wave devices, pp. 214. Prentice-Hall, Englewood Cliffs (1986).

27. M. A. Topinka, B. J. LeRoy, S. E. J. Shaw, E. J. Heller, R. M. Westervelt, K. D. Maranowski, and A. C. Gossard, Science 289, 2323 (2000).

28. M. A. Topinka, B. J. LeRoy, R. M. Westervelt, S. E. J. Shaw, R. Fleischmann, E. J. Heller, K. D. Maranowski, and A. C. Gossard, Nature 410, 183 (2001).

29. J. H. Davies and J. A. Nixon, Phys. Rev. B 39, 3423 (1989).

30. J. A. Nixon and J. H. Davies, Phys. Rev. B 41, 7929 (1990).

31. K. Gloos, P. Utko, M. Aagesen, C. B. Sørensen, J. Bindslev Hansen, and P. E. Lindelof, Phys. Rev. B 73, 125326 (2006).

32. P. Utko, Ph.D. thesis, University of Copenhagen, Copenhagen (2005). 